\title{
Caspase-Independent Pathway is Related to Nilotinib Cytotoxicity in Cultured Cardiomyocytes
}

\author{
Qinghui Yang ${ }^{\mathrm{a}}$ Chunhui Zhang ${ }^{\mathrm{b}}{\text { Hong Wei }{ }^{\mathrm{a}} \text { Zenghui Meng }}^{\mathrm{a}}$ \\ Guangnan $\mathrm{Li}^{\mathrm{a}}$ Yuanyuan $\mathrm{Xu}^{\mathrm{a}}$ Yanjun Chen ${ }^{\mathrm{c}}$ \\ aDepartment of Cardiology, the Fourth Affiliated Hospital of Harbin Medical University, Harbin, \\ ${ }^{b}$ Department of Gastrointestinal Medical Oncology, the Affiliated Tumor Hospital of Harbin Medical \\ University, Harbin, 'Department of Cardiology, Peking University Shenzhen Hospital, Shenzhen, China
}

\section{Key Words}

H9c2 • Autophagy • Cathepsin B • Mitochondria • Apoptosis • Atg5 • LC3 • AIF

\begin{abstract}
Background/Aims: Cardiotoxicity is a predominant side-effect of nilotinib during chronic myeloid leukemia treatment. The underlying molecular mechanism remains unclear. The role of autophagy and mitochondrial signaling was investigated in nilotinib-treated cardiac $\mathrm{H} 9 \mathrm{C} 2$ cells. Methods: Cytotoxicity was assessed using Cell Death Detection kit. Immunoblot and immunofluorescence staining was performed, and cathepsin B and caspase 3 activity was assessed in nilotinib-treated $\mathrm{H} 9 \mathrm{C} 2$ cells with or without distinct pathway inhibitor or specific siRNA. Results: Nilotinib time- and dose-dependently induced H9C2 apoptosis, which was not completely prevented by the pan caspase inhibitor z-VAD-fmk. Following nilotinib treatment, mitochondrial membrane potential decreased significantly accompanied with remarkable morphological changes. Nuclear translocation of mitochondrial apoptosis inducing factor (AIF) and increased p53 was detected in nilotinib-treated cells. AIF knockdown prevented nilotinib-induced increase of p53 and apoptosis. Additionally, increased cathepsin B activity was detected, and inhibition of cathepsin B by CA-074Me prevented nilotinib-induced apoptosis and nuclear translocation of AIF. Moreover, increased Atg5 and transition of LC3-I to LC3-II was revealed following nilotinib treatment. Increased cathepsin B activity and apoptosis by nilotinib was significantly prohibited by specific autophagy inhibitor bafilomycin $A$ and Atg5 knockdown. Conclusion: Our findings demonstrate that nilotinib increases autophagy and cathepsin B activity, leading to mitochondrial AIF release and nuclear translocation, which is responsible for p53 and apoptosis induction in $\mathrm{H} 9 \mathrm{C} 2$ cells.
\end{abstract}

Q. Yang and C. Zhang equally contribute to this work.

Yanjun Chen, MD

KARGER
Department of Cardiology, Peking University Shenzhen Hospital

No1120, Lianhua Road, Futian District, Shenzhen (China)

Tel. +86755 83923333-6334, E-Mail chenyanjunhyd@163.com 


\section{Introduction}

Chronic myeloid leukemia (CML), a very common hematological malignity, is predominately caused by a reciprocal chromosomal translocation between chromosome 9 and 22 leading to a new gene formation of $B C R-A B L$ [1]. The new fusion gene encodes a constitutively active tyrosine kinase that allows the myeloid cells to proliferate out of control [1].

Nilotinib, one of the second-generation of BCR-ABL tyrosine kinase inhibitor (TKI), is widely used in clinic to enhance efficacy and reduce resistance during CML treatment $[2,3]$. Nevertheless, due to the potential interference of other tyrosine kinase signaling pathways, the TKI including nilotinib often results in various side-effects such as neutropenia, thrombocytopenia, and cardiotoxicity in CML patients [2-4]. Generally, the cardiotoxicity resulting from TKI manifests multifocal cardiomyocyte necrosis and/or apoptosis, myocardial fibrosis, myocardial hypertrophy and dilatation, and QT interval prolongation [5]. It was reported that nilotinib can increase generation of reactive oxygen species (ROS) and activation of caspase3/7 in cultured human cardiomyocytes [6]. Using rat cardiac H9C2 cells, nilotinib showed toxicity under clinically relevant concentrations, such as mitochondrial dysfunction, increased ROS production and endoplasmic reticulum (ER) stress induction, as well as increased caspase $3 / 7$ and apoptosis [7]. In addition, it was proved that the cytotoxicity of doxorubicin, a very commonly used anti-cancer chemical reagent, was significantly enhanced by nilotinib [8]. And importantly, a disproportionate increase of doxorubicinol was found in cardiac tissue with more severe necrosis and vacuole formation [8]. To decrease and/or eliminate TKI cardiotoxicity, the underlying mechanisms have been investigated but still remain unclear. Further detailed researches are highly required for reassessing the efficacy and risk of the TKI in order to prevent their side-effects including cardiotoxicity.

In this study, we explored the potential mechanisms of nilotinib-mediated cardiotoxicity using H9C2 cells by focusing on the role of autophagy and mitochondrial function in apoptosis induction. Our findings demonstrate that autophagy and cathepsin B activity is significantly increased in nilotinib-treated $\mathrm{H} 9 \mathrm{C} 2$ cells, leading to mitochondrial apoptosis inducing factor (AIF) release and nuclear translocation, which is responsible for p53 and apoptosis induction.

\section{Materials and Methods}

\section{Cell culture and treatment}

Primary rat neonatal cardiomyocytes were isolated from 2-day-old Sprague-Dawley rat heart as reported previously [9]. Rat cardiac H9C2 cells purchased from ATCC were cultured in DMEM media supplemented with $10 \%$ fetal calf serum and $100 \mathrm{U} / \mathrm{ml}$ penicillin/streptomycin (Gibco). When reached $70 \%$ confluences, cells were treated with $0,1,5$, and $10 \mu \mathrm{M}$ of nilotinib (Sigma) for various time periods $(0$, $12,24$, and $48 \mathrm{~h})$. In experiments using pan caspase inhibitor z-VAD-fmk (50 $\mu \mathrm{M})$, cathepsin B inhibitor CA-074Me $(10 \mu \mathrm{M})$, and autophagy inhibitor bafilomycin A1 (5 nM), cells were pretreated for $1 \mathrm{~h}$ with the indicated inhibitor followed by nilotinib administration in the presence of inhibitor.

\section{Knockdown assay}

The following validated siRNAs were used for rat AIF and Atg 5 knockdown, respectively [10, 11]. siAIF: 5'-cauagaagcaugcagcaucuccugc-3'; siAtg5: 5'-gguuuggacgaauuccaacuuguuu-3'. Universal Negative Control siRNA was used as the control for non-sequence-specific effects. Transfection was performed at a final concentration of $50 \mathrm{nM}$ using Lipofectamine RNAiMAX (RNAiMAX : siRNA = $1 \mu \mathrm{l}: 10 \mathrm{pmol}$ ) according to the manufacturer's instructions (Invitrogen). After $48 \mathrm{~h}$, total cellular protein was extracted and the protein level of AIF and Atg5 was assessed using immunoblot assay.

\section{KARGER}




\section{Cellular Physiology Cell Physiol Biochem 2017;42:2182-2193

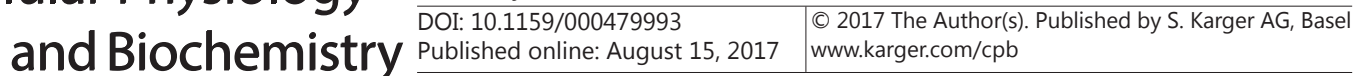

\section{Cell Death Detection ELISA}

The level of apoptotic cells was determined using the Cell Death Detection ELISA kit (Roche) according to the manufacturer's protocol. Briefly, $20 \mu \mathrm{l}$ of cytoplasmic fractions from $1 \times 10^{4}$ cells were applied to the wells of the MP-modules that have been coated by anti-histone antibody, followed by adding $180 \mu \mathrm{l}$ of Incubation Buffer. Of note, the wells containing $200 \mu \mathrm{l}$ of Incubation Buffer were used as the blank control. The plates were kept for $90 \mathrm{~min}$ on a shaker at room temperature. After 3 washes with $250 \mu \mathrm{l}$ of Washing Buffer, $100 \mu \mathrm{l}$ of Conjugate Solution containing anti-DNA-peroxidase was added to each well, and incubated for $90 \mathrm{~min}$. After 3 washes, $100 \mu \mathrm{l}$ of Substrate Solution containing ABTS (2, 2'-azino-di-[3ethylbenzthiazoline sulfonate]) was added to each well, and incubated for $10 \mathrm{~min}$ on a plate shaker until the expected color for the photometric analysis emerged. Each well was read at a wavelength of $405 \mathrm{~nm}$. The value from blank control was subtracted from all samples. Three independent duplicate experiments were performed. The subtracted value of OD405 was used to indicate the level of apoptotic cells.

\section{Caspase3 Activity Detection Assay}

Caspase3, the family of cysteine proteases, exists in cells as an inactive $32 \mathrm{kDa}$ proenzyme. While apoptosis occurs, caspase 3 is cleaved into active 17 and $12 \mathrm{kDa}$ subunits by various upstream proteases such as caspase6, caspase8, and Granzyme B [12]. Caspase3 activity was assessed using the Caspase3 Colorimetric Protease Assay (Invitrogen), by recognizing and quantitating $p$ NA-conjugated amino acid sequence DEVD that is localized the upstream of the capsase 3 cleavage site in its substrate PARP. Briefly, 50 $\mu \mathrm{l}$ of cytosol extract containing $25 \mu \mathrm{g}$ protein each sample was added to the wells of 96 -well plate, followed by addition of $50 \mu \mathrm{l}$ of $2 \mathrm{x}$ Reaction Buffer/10mM DTT. Thereafter, $5 \mu \mathrm{l}$ of $4 \mathrm{mM}$ DEVD- $p$ NA substrate was added and incubated at $37^{\circ} \mathrm{C}$ for $2 \mathrm{~h}$. The absorbance of $p \mathrm{NA}$ at $400 \mathrm{~nm}$ from apoptotic cells were recorded in a microplate reader. Three independent duplicate experiments were performed. Fold increase over nontreated control was used for comparison of caspase 3 activity.

\section{Western blot}

Cells were washed 3 time with cold phosphate-buffered saline (PBS), and then lysed in RIPA buffer [50 mM Tris-HCl pH 7.4, 1\% NP-40, 0.25\% DOC, $150 \mathrm{mM} \mathrm{NaCl}, 1 \mathrm{mM}$ EDTA, $2 \mathrm{mM} \mathrm{Na}_{3} \mathrm{VO}_{4}, 5 \mathrm{mM} \mathrm{NaF}$, and $1 \mathrm{x}$ protease inhibitor cocktail (Sigma)] for $30 \mathrm{~min}$ on ice. The cell lysates were centrifuged at $4^{\circ} \mathrm{C}$ for 20 $\min$ at 12, $500 \mathrm{rpm}$, and the supernatant was then collected. In addition, mitochondrial protein and nuclear protein were isolated using Mitochondria Isolation Kit (ThermoFisher) and Nuclear Extraction Kit (Abcam), respectively. Samples containing equal amounts of proteins determined by BCA assay (Bio-Rad) were subjected to 4-15\% SDS-polyacrylamide gel electrophoresis and transferred to nitrocellulose membranes (GE Healthcare Bioscience). The blots were incubated with the indicated primary antibodies in cold room for overnight. Following 3 washes with TTBS, the membranes were incubated for $1 \mathrm{~h}$ with HRP-conjugated goat anti-mouse or rabbit IgG (Invitrogen, 1:5000). The enhanced chemiluminescence reagents (Invitrogen) were applied to develop the signal. The primary antibodies includes rabbit anti-caspase3 (Abcam, 1:1000), rabbit anti-cleaved caspase3 (Abcam, 1:200), rabbit anti-AIF (Abcam, 1:500), mouse anti-HSP60 (Thermo, 1:1000), mouse anti-Histone H3 (Abcam, 1:500), mouse anti-p53 (Cell Signaling, 1:1000), rabbit anti-Atg5 (Abcam, 1:400), mouse anti-LC3 antibody (Abcam, 1:600), and mouse anti-GAPDH (Sigma, 1:2000).

\section{JC-1 staining assay}

JC-1, a cationic dye, exhibits potential-dependent accumulation/aggregates in mitochondria (Abcam). The level of mitochondria membrane potential (MMP) was determined using JC-1 stain by comparing the ratio of J-aggregates to monomers. Briefly, $2 \times 10^{5}$ cells each condition were collected and washed once in 1x Dilution Buffer, followed by staining with $2 \mu \mathrm{M}$ of JC- 1 in $1 \mathrm{x}$ Dilution Buffer for $30 \mathrm{~min}$ at $37^{\circ} \mathrm{C}$. The absorbance of $590 \mathrm{~nm}$ and $520 \mathrm{~nm}$ was measured in a microplate reader, and the ratio was calculated and compared. Three independent duplicate experiments were performed.

\section{Cathepsins activity detection}

Cathepsin B, D, and L activity was detected in $50 \mu \mathrm{l}(50 \mu \mathrm{g})$ lysates from $2 \times 10^{6}$ cells using a fluorescencebased assay according to the manufacturer's guidelines (Abcam). $50 \mu \mathrm{l}$ Lysis Buffer alone was used as the background control. Briefly, for cathepsin B activity detection, $50 \mu \mathrm{l} \mathrm{CB}$ Reaction Buffer was added to each well and $2 \mu \mathrm{l}$ of $10 \mathrm{mM} \mathrm{CB}$ substrate Ac-RR-AFC was then added. The reaction was incubated at $37^{\circ} \mathrm{C}$ for 


\section{Cellular Physiology Cell Physiol Biochem 2017;42:2182-2193 \\ \begin{tabular}{ll|l} 
DOI: 10.1159/000479993 & O 2017 The Author(s). Published by S. Karger AG, Basel \\
www.karger.com/cpb
\end{tabular}}

Yang et al.: Nilotinib Induced Caspase-Independent Signaling in H9C2

$1 \mathrm{~h}$. Thereafter, fluorescence signal was measured using microplate reader at $\mathrm{Ex} / \mathrm{Em}=400 / 505 \mathrm{~nm}$. The background control was subtracted from sample readings. The relative fluorescence unit (RFU) was used to indicate cathepsin B activity. Data were obtained from three independent duplicate experiments.

\section{Immunofluorescence staining}

Mitochondria morphology was revealed using MitoTracker Red CMXRos (Invitrogen) in live H9C2 cells cultured on coverslips according to the manufacturer's procedure. Briefly, $0.25 \mu \mathrm{M}$ of MitoTracker was applied to cells and incubated at $37^{\circ} \mathrm{C}$ for $20 \mathrm{~min}$. For AIF staining, cells were then fixed in $4 \%$ PFA/PBS for $15 \mathrm{~min}$ and permeabilized for $10 \mathrm{~min}$ in $0.25 \%$ TritonX-100/PBS solution. Rabbit anti-AIF antibody (1:100 diluted in 5\%BSA/PBS, Abcam) was applied for $1 \mathrm{~h}$ at room temperature. After 3 washes with PBS, AlexaFluo 488-conjugated goat anti-rabbit IgG (1:2000 diluted in 5\%BSA/PBS, Invitrogen) were incubated for $45 \mathrm{~min}$ at room temperature. After 3 washes, cells were mounted on glass slide with antifade media (Invitrogen). Images were taken using laser scanning confocal microscopy system (Zeiss).

\section{Statistical analysis}

Data are shown as mean \pm SD. Statistical analysis was performed with GraphPad Prism 6. Oneway ANOVA and unpaired $t$-test was used for multiple groups' comparison and two groups' comparison, respectively. The $p$ value equal to or less than 0.05 was considered as significant differences.

\section{Results}

Nilotinib induced apoptosis is caspase-independent in $\mathrm{H} 9 \mathrm{C} 2$ cells

Nilotinib, the second-generation tyrosine kinase inhibitor, is mainly used in clinic for the treatment of CML $[1,3,4]$. However, various side-effects occur during nilotinib treatment, including neutropenia, thrombocytopenia and cardiotoxicity [2, 4]. Here, we investigated the mechanism by which nilotinib causes cardiomyocyte injury in cultured H9C2 cell, a sub-clone derived from embryonic rat heart tissue. Nilotinib-induced cytotoxicity was analyzed by evaluating the level of apoptotic cells using the Cell Death Detection ELISA Kit. The level of cell death/apoptosis is presented as the absorbance of anti-DNA-peroxidaseinduced substrate reactions at $405 \mathrm{~nm}$. Following $10 \mu \mathrm{M}$ of nilotinib treatment, apoptosis was increased significantly $(p<0.01)$ since $12 \mathrm{~h}(0.091 \pm 0.018)$, persisting to $24 \mathrm{~h}(0.165$ $\pm 0.011)$ and $48 \mathrm{~h}(0.265 \pm 0.004)$ in comparison with $0 \mathrm{~h}(0.041 \pm 0.007)$ in H9C2 cells (Fig. 1A), showing a time-dependent pattern. We also treated H9C2 cells for $24 \mathrm{~h}$ with a variety of doses of nilotinib. Our data show that compared to $0 \mu \mathrm{M}(0.040 \pm 0.007)$, cell death level increased significantly $(p<0.05)$ in nilotinib treated cells at $5 \mu \mathrm{M}(0.083 \pm 0.010)$ and $10 \mu \mathrm{M}(0.177 \pm 0.012)$, presenting a dose-dependent manner (Fig. 1B). Furthermore, quantitative result from immunoblot assay reveals that the cleaved caspase3 level increased significantly in nilotinib treated cells (Fig. 1C). Although caspase is a major cellular apoptosis pathway in a numerous types of cells [11], other signaling pathway may also be involved in nilotinib-induced $\mathrm{H} 9 \mathrm{C} 2$ injury [13]. In cultured $\mathrm{H} 9 \mathrm{C} 2$ and primary cardiomyocytes, we inhibited nilotinib-induced caspase 3 activation by using $50 \mu \mathrm{M}$ of z-VAD-fmk, a pan caspase inhibitor (Fig. 1C,D), and explored the effect of caspase3 inhibition on nilotinib-mediated H9C2 cytotoxicity. We found that nilotinib-induced H9C2 apoptosis was not thoroughly prevented (Nil+ZAD: $0.111 \pm 0.013$ vs Nil: $0.184 \pm 0.014$ and CTL: $0.053 \pm 0.005, p<0.01$ ) by ZAD although caspase3 activation was totally blocked (Fig. 1C-E). Similar results were also obtained from nilotinib treated primary cardiomyocytes (Fig. 1E). These findings suggest that caspase-independent signaling pathway may, at least partially, be involved in nilotinibinduced H9C2 injury.

\section{Mitochondrial dysfunction is related to nilotinib-induced H9C2 injury}

Mitochondria play a crucial role in apoptosis induction in mammalian cells including myocyte $[14,15]$. It was reported that reduction of mitochondrial membrane potential (MMP) is related to the onset of apoptosis $[16,17]$. In this study, MMP was assessed using 
Fig. 1. Nilotinib-induced apoptosis is caspase-independent in $\mathrm{H} 9 \mathrm{C} 2$ cells.A and B. H9C2 cells were treated with nilotinib. Time- (A) and concentrationdependent (B) cellular apoptosis was assessed using the Cell Death Detection ELISA Kit. C. H9C2 cells were pretreated for $1 \mathrm{~h}$ with $50 \mu \mathrm{M}$ of the pan caspase 3 inhibitor z-VAD-fmk, followed by treatment with $10 \mu \mathrm{M}$ of nilotinib for $24 \mathrm{~h}$ in the presence of VAD. Non-treated cell was used as control. Total cellular protein was isolated using RIPA buffer, and immunoblot assay was performed to measure the cleaved caspase3 level. D and E. Primary cardiomyocytes and H9C2 cells were pretreated for $1 \mathrm{~h}$ with $50 \mu \mathrm{M}$ of the pan caspase 3 inhibitor $\mathrm{z}^{-}$ VAD-fmk, followed by treatment with 10 $\mu \mathrm{M}$ of nilotinib for $24 \mathrm{~h}$ in the presence of VAD. Non-treated cell was used as control. The activity of caspase 3 was assessed using the Caspase 3 Colorimetric Protease Assay (D). Effects of VAD on apoptosis were investigated in nilotinibtreated H9C2 cells (E). CTL: control, Nil: nilotinib, casp3: caspase3. Data are from three independent duplicate experiments (A, B, D, and E). Representative blot was provided in C. A: * $\mathrm{p}<0.05$ vs 0 h, \# p<0.01 vs 12 h, $\$$ p < 0.01 vs $24 \mathrm{~h}$; B: * $\mathrm{p}<0.05$ vs $0 \mu \mathrm{M}, \# \mathrm{p}<0.05$ vs $1 \mu \mathrm{M}, \$$ $\mathrm{p}<0.001$ vs $5 \mu \mathrm{M}$; C - E: * $<<0.001$ vs CTL, \# p $<0.001$ vs Nil.

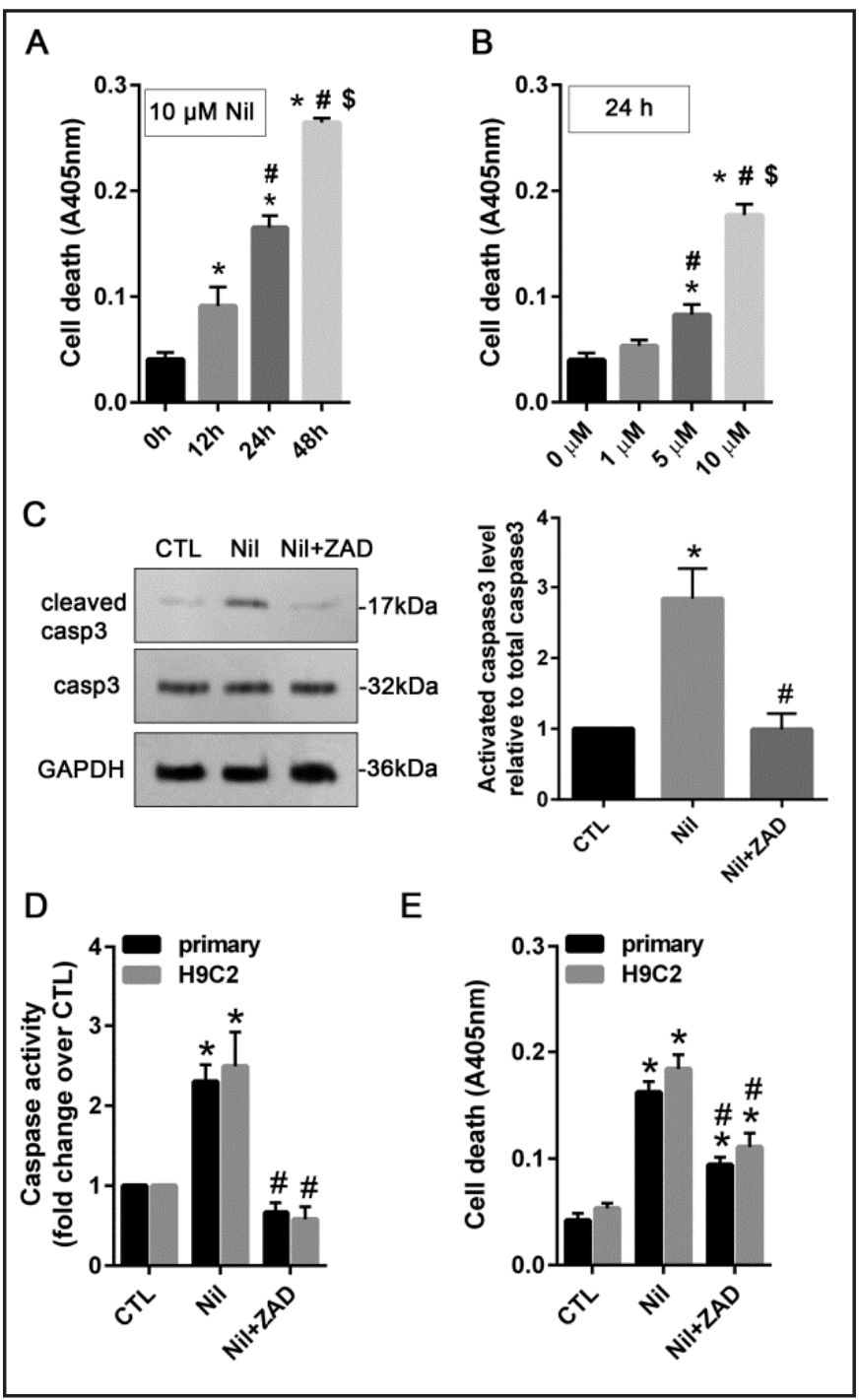

JC-1 assay. In nilotinib treated cells, JC-1 assay shows that MMP was significantly decreased $(1.898 \pm 0.144$ vs. $0.102 \pm 0.040, p<0.001)$ as compared with non-treated H9C2 cells (Fig. 2A). Apoptosis inducing factor (AIF), belonging to the FAD-dependent oxidoreductase family, is localized to mitochondrial intermembrane space and has a dual role in controlling cellular survival and death $[18,19]$. During apoptosis, mitochondrial AIF is found to be released to cytosol and a proteolytic processing thus occurs at amino-acid position 101 leading to the mature form production, a soluble AIF protein $[18,19]$. In response to specific death signals, soluble AIF is translocated to the nucleus, where it induces DNA damage and apoptosis in a caspase-independent manner $[18,19]$. To elucidate the role of AIF, we prepared total cellular proteins as well as mitochondrial and nuclear fractions from nilotinib-treated H9C2 cells, and analyzed the level of AIF in different fractions (Fig. 2B). The specificity of extracted mitochondrial and nuclear fraction was indicated by the abundance of heat shock protein 60 (HSP60, a mitochondrial protein marker) and histone H3 (a nuclear fraction marker), respectively. Compared to control, total AIF abundance increased obviously in nilotinib treated cells (Fig. 2B1). Further immunoblot assay from mitochondrial fractions showed that the increased AIF by nilotinib was mainly localized in mitochondria (Fig. 2B2). Moreover, the amount of AIF in nuclear fraction increased remarkably in nilotinib treated cells (Fig. 2B3). These results indicate the translocation of AIF from mitochondria to nucleus. We also stained mitochondria using MitoTracker and then performed AIF staining. 


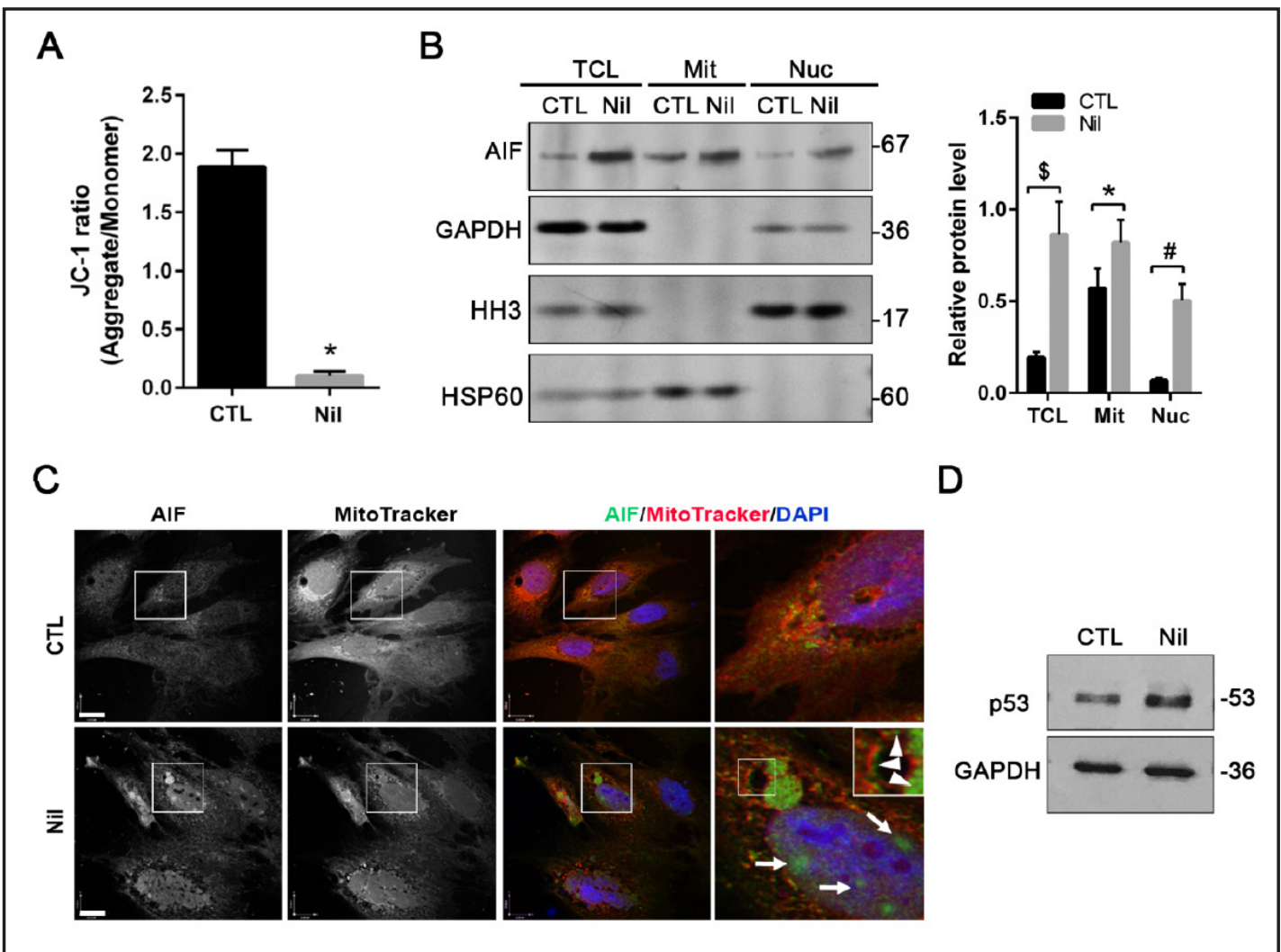

Fig. 2. Mitochondrial dysfunction occurs in nilotinib-treated H9C2 cells.H9C2 cells were treated for $24 \mathrm{~h}$ with $10 \mu \mathrm{M}$ of nilotinib. A. Mitochondria membrane potential (MMP) was evaluated using JC-1 assay by comparing the ratio of aggregate/A590 $\mathrm{nm}$ to monomer/A520 nm. Data are from three independent duplicate experiments. ${ }^{*} \mathrm{p}<0.001$ vs CTL. B. To assess the level of AIF, total cellular protein, mitochondrial protein, and nuclear protein was extracted, respectively. The abundance of AIF was measured using immunoblot assay. ${ }^{*} \mathrm{p}<0.05,{ }^{\#} \mathrm{p}<0.01,{ }^{\$} \mathrm{p}<0.001$. C. mitochondria was revealed using MitoTracker in live cells. Cells were then fixed and permeabilized for AIF staining. Arrow: nuclear AIF, arrowhead: cytosolic AIF. Bar $=15 \mu \mathrm{M}$. D. The level of p53 was assessed using immunoblot of total cellular extraction. B and D: Three independent experiments were performed and representative blots were provided. CTL: control, Nil: nilotinib, TCL: total cellular protein, Mit: Mitochondria protein, Nuc: Nuclear extraction, AIF: apoptosis inducing factor; HH3: histone H3; HSP60: Heat shock protein 60.

Following nilotinib treatment, mitochondrial morphology changed dramatically, showing a distorted and irregular shape (Fig. 2C). Additionally, AIF did not show well co-localization with MitoTracker signals in nilotinib-treated cells, and nuclear AIF signal was much more obvious after nilotinib treatment (Fig. 2C), suggestive of a release of AIF from mitochondria to cytosol and then to nuclei.

Following DNA damage, induction of caspase-independent p53 signaling also initiates apoptosis $[20,21]$. Immunoblot assay shows that compared to control the p53 protein level increased significantly in nilotinib-treated H9C2 cells (Fig. 2D). Based on this finding, we proposed a hypothesis that overproduced p53 after nilotinib treatment may result from the translocated nuclear AIF. To validate this hypothesis, we performed siRNA knockdown assay in H9C2 cells to silent AIF expression. Our results show that AIF protein level was dramatically decreased in nilotinib-treated cells, and that nilotinib-induced upregulation of p53 was remarkably prevented by AIF knockdown (Fig. 3A). Moreover, induction of apoptosis after nilotinib treatment was also decreased in AIF knockdown cells (Fig. 3B). Accordingly, these findings demonstrate that mitochondrial dysfunction is associated to nilotinib induced cytotoxicity possibly through the AIF/p53 pathway.

\section{KARGER}


Fig. 3. AIF knockdown protects $\mathrm{H} 9 \mathrm{C} 2$ cells from nilotinib toxicity.The siRNA targeting rat AIF were introduced into $\mathrm{H} 9 \mathrm{C} 2$ cells using RNAiMAX. $24 \mathrm{~h}$ later, the cells were treated for $24 \mathrm{~h}$ with $10 \mu \mathrm{M}$ of nilotinib. A. Immunoblot assay was performed for AIF and p53 level. Three independent experiments were performed and representative blot was provided. B. Effects of AIF knockdown on nilotinib-induced apoptosis was evaluated using the Cell Death Detection

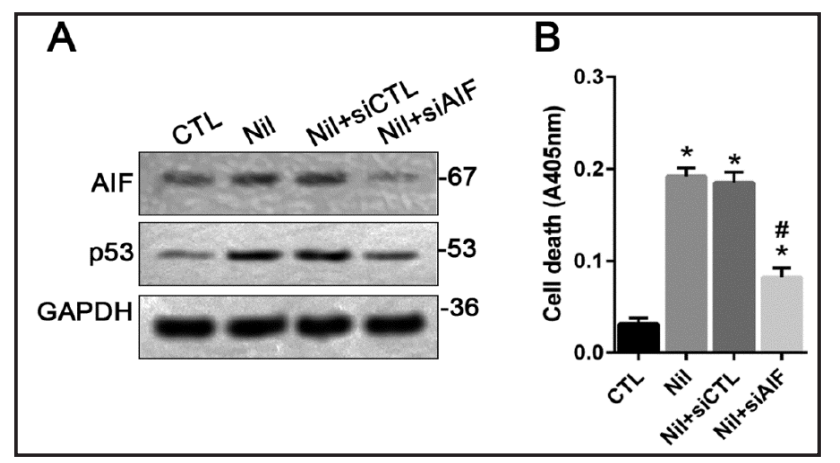
ELISA Kit. Data are from three independent duplicate experiments. ${ }^{*} \mathrm{p}<0.05$ vs CTL, $\#$ p $<0.05$ vs Nil. CTL: control, Nil: nilotinib, siCTL: Control siRNA, siAIF: siRNA targeting rat AIF, AIF: apoptosis inducing factor.

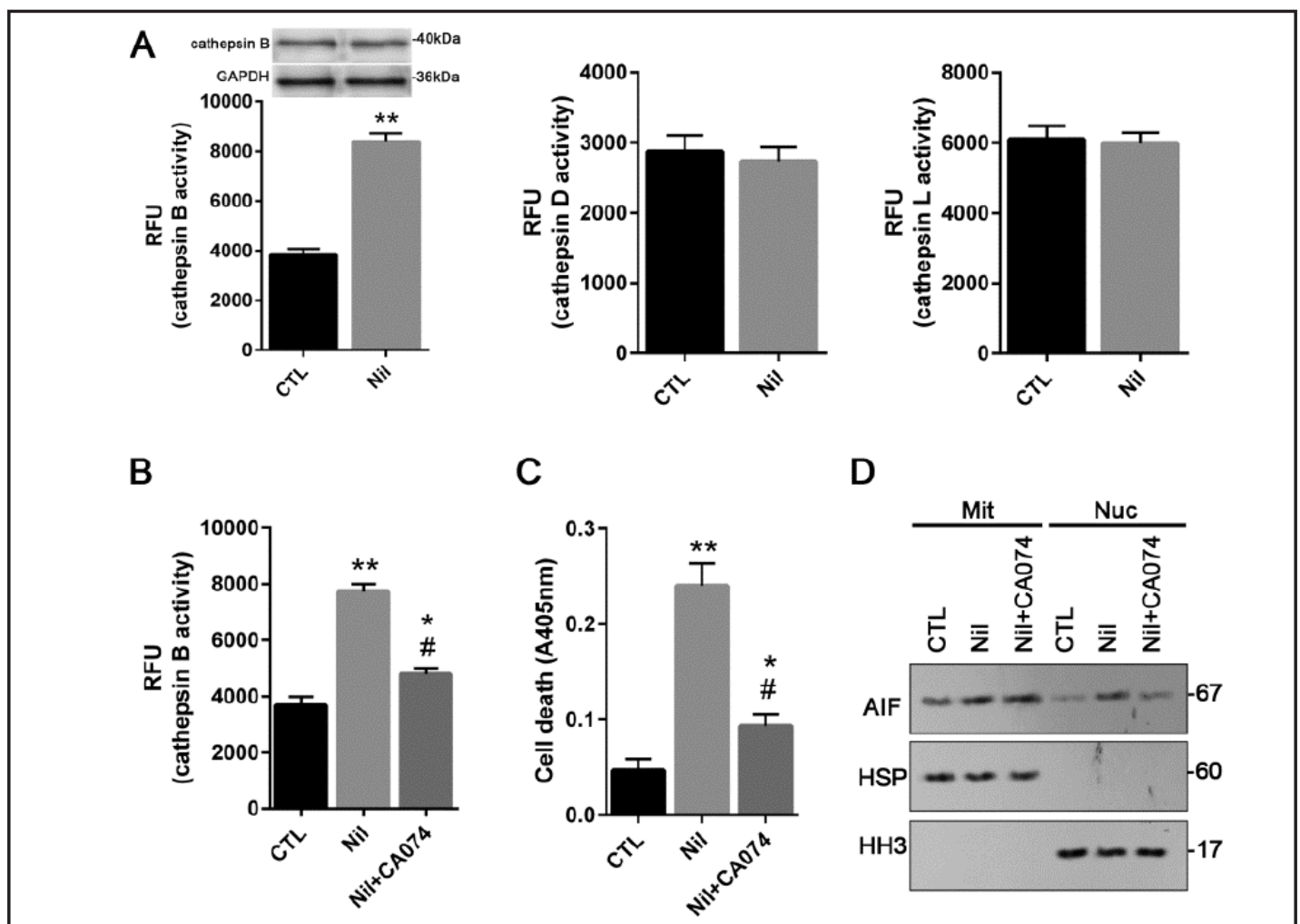

Fig. 4. Over-activated cathepsin B is responsible for mitochondrial AIF release and cell death in nilotinibtreated H9C2 cells.A. H9C2 cells were treated for $24 \mathrm{~h}$ with $10 \mu \mathrm{M}$ of nilotinib. Total cellular protein was extracted from $2 \times 10^{6}$ cells, and $50 \mu$ g of lysates was used for assessment of cathepsin B, D, and L activity. Immunoblot assay was used for evaluation of total cathepsin B protein level. B - E. H9C2 cells were pretreated for $45 \mathrm{~min}$ with $10 \mu \mathrm{M}$ of cathepsin B inhibitor CA-074Me, and then treated for $24 \mathrm{~h}$ with $10 \mu \mathrm{M}$ of nilotinib in the presence of CA-074Me. Total cellular protein was isolated and cathepsin B activity was determined (B). Effect of CA-074Me on nilotinib-induced apoptosis was evaluated using the Cell Death Detection ELISA assay (C). Mitochondrial and nuclear protein was extracted, and immunoblot assay was performed for the abundance of AIF (D). A - C: Data were obtained from three independent duplicate experiments. D. Representative blot was from 3 independent experiments. ${ }^{*}$ p $<0.01$ vs CTL, ${ }^{* *}$ p $<0.001$ vs CTL, \# p $<0.01$ vs Nil. CTL: control, Nil: nilotinib, RFU: relative fluorescence unit, Mit: Mitochondria protein, Nuc: Nuclear extraction, AIF: apoptosis inducing factor, HSP: Heat shock protein 60, HH3: Histone H3.

Over-activated cathepsin B leads to nilotinib-induced mitochondrial AIF release

Cathepsins, the lysosomal cysteine proteases, localize in lysosomes and/or endosomes, and function to degrade endocytosed or unwanted proteins [22]. Based on the type of 
Fig. 5. Increased autophagy activity is detected in nilotinibtreated H9C2 cells. H9C2 cells were treated for $24 \mathrm{~h}$ with 10 $\mu \mathrm{M}$ of nilotinib. Total cellular protein was extracted, and immunoblot assay was performed for the expression of Atg5 (A) and LC3 (B). Experiments were performed in triplicate, and representative blot was shown.*
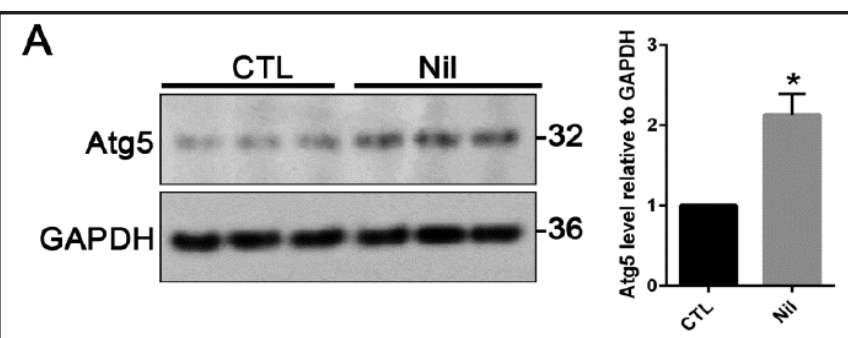

B

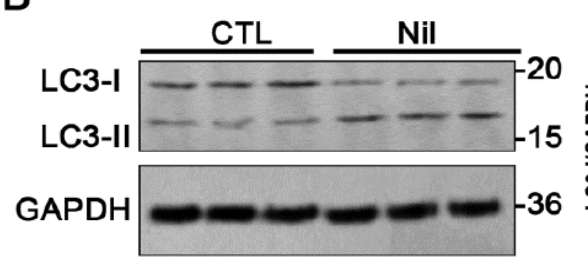

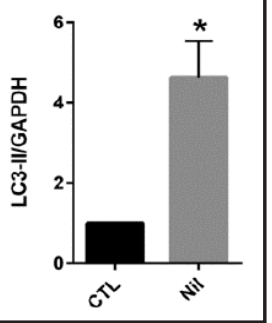

p<0.01 vs CTL. CTL: control, Nil: nilotinib. Atg5: autophagy protein 5. LC3: microtubule-associated protein 1 light chain 3.

protease substrates, cathepsins are classified as i) the serine proteases cathepsins $\mathrm{A}$ and $\mathrm{G}$, ii) the aspartic proteases cathepsins $\mathrm{D}$, and iii) $\mathrm{E}$, and the lysosomal cysteine cathepsins including B, C, F, H, K, L, O, S, V, X and W [22]. Cathepsin B, one of the major lysosomal cysteine proteases, is widely expressed in mammalian cells and plays a critical role in apoptosis induction [23]. In this study, we analyzed the activity level of cathepsin B, D and L in nilotinib-treated H9C2 cells. Compared to control, the activity of cathepsin B increased significantly $(p<0.01)$ following nilotinib treatment, whereas the activity of cathepsin D and L showed no significant change (Fig. 4A). Of note, total cathepsin B protein level showed no change following nilotinib treatment (Fig. 4A). A specific cathepsin B inhibitor CA074 was applied to address the role of cathepsin B over-activation in nilotinib cytotoxicity. Our data show that increased cathepsin B activity by nilotinib was dramatically inhibited by CA074 (Fig. 4B). As expected, nilotinib induced apoptosis was significantly prevented by CA074 (Fig. 4C). Furthermore, immunoblot assay shows that nilotinib-induced increase of AIF in nuclei was remarkably prevented by CA074 (Fig. 4D). Therefore, these findings suggest that over-activated cathepsin B is related to nilotinib-mediated $\mathrm{H} 9 \mathrm{C} 2$ cytotoxicity potentially by inducing nuclear translocation of mitochondrial AIF.

Blockage of autophagy prevents cathepsin B over-activation and cytotoxicity in nilotinibtreated H9C2 cells

Autophagy, a lysosome-mediated intracellular degradation pathway, functions both as a pro-survival and a pro-apoptosis mechanism depending on the stimuli [24-26]. It was reported that activation of autophagy is implicated in oxidative stress, ischemia/reperfusion, or high-glucose injured cardiomyocytes [27-29]. The process of autophagy is controlled by numerous autophagy-related proteins [24]. Autophagy gene (Atg5), an E3 ubiquitin ligase, is required for autophagy function due to its role in autophagosome elongation by forming a complex with Atg12 and Atg16L1 [26]. This complex is required for the transition of LC3-I to LC3-II [26]. Compared to control, the abundance of Atg5 increased remarkably in nilotinib treated H9C2 cells (Fig. 5A). In consistent, the amount of LC3-I decreased whereas LC3II increased following nilotinib treatment (Fig. 5B), indicating that autophagy function is increased and may be involved in nilotinib-mediated cardiomyocyte toxicity.

To validate the role of over-activated autophagy in nilotinib-induced $\mathrm{H} 9 \mathrm{C} 2$ injury, autophagy function was disrupted by bafilomycin A (BFA), an inhibitor of autophagosomelysosome fusion. To specifically inhibit autophagy function, Atg5 knockdown assay was performed in this study. Our data show that both BFA and Atg5 knockdown prohibited the 
Fig. 6. Blockage of autophagy prevents cathepsin B activity and nilotinib toxicity in H9C2 cells.H9C2 cells were pretreated for $1 \mathrm{~h}$ with $5 \mathrm{nM}$ of autophagy inhibitor Bafilomycin A (BFA), followed by 24 h-incubation with $10 \mu \mathrm{M}$ of nilotinib in the presence of BFA. Or, the siRNA targeting rat Atg5 were introduced into $\mathrm{H} 9 \mathrm{C} 2$ cells with RNAiMAX. $24 \mathrm{~h}$ later, the cells were treated for $24 \mathrm{~h}$ with $10 \mu \mathrm{M}$ of nilotinib. A. Total cellular protein was extracted, and immunoblot assay was performed for expression of LC3 and Atg5, respectively. 3 independent experiments were performed, and the representative blot was shown. ${ }^{*} \mathrm{p}<0.001$ vs CTL. \# p<0.001 vs Nil+siCTL. B. Cathepsin B activity was evaluated using a fluorescence-based assay. C. Effect of BFA and siAtg5 on nilotinib-induced apoptosis was evaluated using the Cell Death Detection ELISA assay. CTL: control. Nil: nilotinib. BFA: Bafilomycin A. Atg5: autophagy protein 5. LC3: microtubule-associated protein 1 light chain 3. B and C: experiments were performed in triplicate. * p $<0.05$ vs CTL, ${ }^{* *}$ p $<0.01$ vs CTL, ${ }^{* * *}$ p $<0.001$ vs CTL. \# $p<0.05$ vs Nil or Nil+siCTL.

transition of LC3-I to LC3-II(Fig. 6A), proving that autophagy function was blocked successfully. Following autophagy inhibition, we assessed the activity of cathepsin B in nilotinib treated H9C2 cells. Increased cathepsin B activity by nilotinib treatment was significantly prevented by BFA or siAtg5 (Fig. 6B). Notably, induction of apoptosis was decreased by BFA and Atg 5 knockdown in nilotinib-treated cells (Fig. 6C). These findings demonstrate that autophagy over-activation increases cathepsin B activity, which participates in nilotinib cytotoxicity in H9C2 cells.

\section{Discussion}

It was reported that maximal plasma concentration in nilotinib $(400 \mathrm{mg}$ twice a day) treated patients was about $4.27 \mu \mathrm{M}$ [30]. In this study, H9C2 cells were treated with increasing doses $(0,1,5$ and $10 \mu \mathrm{M})$ of nilotinib for $24 \mathrm{~h}$. We also treated cells with 10 $\mu \mathrm{M}$ of nilotinib for a variety of time periods from 0 to $48 \mathrm{~h}$. The cellular apoptosis was detected for nilotinib cytotoxicity assessment. As previously reported [7], our data show that nilotinib time- and dose- dependently increased cell death in cultured H9C2 cells. In nilotinib treated cells, we also detected increased active caspase 3 using immunoblot assay. It has been identified that increased ROS production plays a key role in nilotinib induced cardiotoxicity $[6,7]$. Induction of intrinsic mitochondrial apoptotic signaling by ROS and mitochondrial DNA damage triggers caspase-dependent or -independent apoptotic pathway by promoting mitochondrial outer membrane permeabilization and thus translocation of cytochrome c and/or AIF from mitochondria to cytosol and/or nuclei $[18,19]$. Accordingly, we hypothesize that caspase-independent pathway may be involved in nilotinib-induced 
cardiomyocyte toxicity. Following nilotinib treatment, mitochondrial membrane potential (MMP) decreased significantly in accompany with dramatic alteration of mitochondrial morphologies, indicating that mitochondrial function was impaired by nilotinib. As expected, nilotinib-induced apoptosis was not completely prevented by a pan caspase inhibitor z-VADfmk in cultured both H9C2 and the primary cardiomyocytes. These findings suggest that caspase-independent intrinsic mitochondrial apoptotic signaling may play a role in the development of nilotinib cardiomyocyte toxicity.

In cultured $\mathrm{H} 9 \mathrm{C} 2$ cells, treatment with doxorubicin, a widely used anti-cancer chemical reagent, results in mitochondrial AIF release and translocation to the nuclei [8,31]. AIF is normally localized within the mitochondrial intermembrane space. Reduction of MMP may lead to rupture of outer mitochondrial membrane, which results in release of mature AIF from mitochondria to cytosol [21]. The cleaved AIF has a nuclear localization signal motif that allows it to translocate to the nucleus where it interacts with DNA leading to chromatin condensation and DNA degradation [21]. In nilotinib treated cells, translocation of AIF from mitochondria to nuclei was confirmed by immunoblot of cell fractions and confocal microscopy in this study. Following DNA damage, induction of caspase-independent p53 signaling promotes apoptosis [20]. In the current study, compared to control, increased p53 level was detected in nilotinib-treated h9C2 cells, which was remarkably prevented by AIF knockdown. Consistently, increased cell death by nilotinib was significantly prohibited in H9C2 cells with AIF deficiency. Therefore, mitochondrial dysfunction occurs and plays an important role in nilotinib-mediated cardiomyocytes toxicity.

Cathepsin B, one of the major lysosomal cysteine proteases, plays a key role in apoptosis [23]. In hyperthermic injury model performed in H9C2 cells, cardiomyocyte apoptosis was obviously induced in accompany with increased cathepsin B activity [32]. We evaluated the activation level of cathepsin B, D, and L in nilotinib-treated H9C2 cells, and found an increase of only cathepsin B activity. We also confirmed that nilotinib-induced increase of cathepsin $B$ activity was not from its increased protein level. Nevertheless, the potential mechanism by which nilotinib induces cathepsin $B$ activation should be further investigated. Inhibition of cathepsin B activity by CA074 prevented nilotinib-induced apoptosis and nuclear translocation of AIF. In SPARC (secreted protein, acidic and rich in cysteine) overexpressed neuroectodermal tumor cells, cathepsin B facilitates autophagy-mediated apoptosis [33]. Autophagy develops as a primary response to stress stimuli and then triggers apoptosis and/or necrosis [24-26]. The process of autophagosome formation is regulated by several autophagy genes (Atg) such as Atg5 and Atg6/Beclin-1 [27]. Meanwhile, autophagy also plays a critical role in promoting cellular survival. In cultured neonatal rat ventricular myocytes, hypoxia induced apoptosis was decreased by exogenous HGF, in which enhanced autophagy may be involved [34]. Inhibition of autophagy by 3-methyladenine and wortmannin or Atg5 knockout blocks cathepsins-tBid-mitochondrial apoptotic signaling pathway via stabilization of lysosomal membrane in ischemic astrocytes [35]. In this study, the increased abundance of Atg5 was revealed in nilotinib-treated H9C2 cells, suggestive of the increased autophagy function. Evaluation of autophagy was further performed through analysis of the reliable autophagosomal marker microtubule-associated protein I light chain 3 (LC3) [27]. Immunoblot analyses of proteins from nilotinib-treated cells revealed a remarkable transition of LC3-I to LC3-II as compared to control. We further explored if autophagy inhibition could affect/prevent cathepsin B activity and cell death. In this study, autophagy signaling was inhibited by the specific autophagy inhibitor bafilomycin A and Atg5 knockdown. In nilotinib-treated H9C2 cells, cathepsin B activity and cellular apoptosis was also significantly reduced by treatment with either bafilomycin A or Atg5 siRNA.

\section{Conclusion}

Taken together, we demonstrate that over-activated cathepsin B facilitates autophagymediated apoptosis through induction of p53 expression by promoting nuclear translocation 


\section{Cellular Physiology Cell Physiol Biochem 2017;42:2182-2193 \begin{tabular}{l|l|l} 
and Biochemistry 10.1159/000479993 & $\begin{array}{l}\text { C } 2017 \text { The Author(s). Published by S. Karger AG, Basel } \\
\text { www.karger.com/cpb }\end{array}$ \\
\hline
\end{tabular}}

Yang et al.: Nilotinib Induced Caspase-Independent Signaling in H9C2

of mitochondrial AIF in nilotinib treated H9C2 cells. This finding helps us further understand the molecular signaling pathways associated to nilotinib-mediated cardiotoxicity. In addition, we provide evidence that would be great useful to develop practicable prevention of this side effect and enhance its efficacy during treatment of CML.

\section{Acknowledgements}

This work is supported by Heilongjiang Postdoctoral Program, China (LBH-Z16126).

\section{Disclosure Statement}

No.

\section{References}

1 Lambert GK, Duhme-Klair AK, Morgan T, Ramjee MK: The background, discovery and clinical development of BCR-ABL inhibitors. Drug Discov Today 2013;18:992-1000.

-2 Damrongwatanasuk R, Fradley MG: Cardiovascular Complications of Targeted Therapies for Chronic Myeloid Leukemia. Curr Treat Options Cardiovasc Med 2017;19:24

3 Pophali PA, Patnaik MM: The Role of New Tyrosine Kinase Inhibitors in Chronic Myeloid Leukemia. Cancer J 2016;22:40-50,

4 Atallah E: Nilotinib cardiac toxicity: should we still be concerned? Leuk Res 2011;5:577-578.

-5 Kerkelä R, Grazette L, Yacobi R, Iliescu C, Patten R, Beahm C, Walters B, Shevtsov S, Pesant S, Clubb FJ, Rosenzweig A, Salomon RN, Van Etten RA, Alroy J, Durand JB, Force T: Cardiotoxicity of the cancer therapeutic agent imatinib mesylate. Nat Med 2006;12:908-916.

-6 Doherty KR, Wappel RL, Talbert DR, Trusk PB, Moran DM, Kramer JW, Brown AM, Shell SA, Bacus S: Multiparameter in vitro toxicity testing of crizotinib, sunitinib, erlotinib, and nilotinib in human cardiomyocytes. Toxicol Appl Pharmacol 2013 272:245-255.

7 Lekes D, Szadvari I, Krizanova O, Lopusna K, Rezuchova I, Novakova M, Novakova Z, Parak T, Babula P: Nilotinib induces ER stress and cell death in H9c2 cells. Physiol Res 2016;65:S505-S514.

-8 Zhou ZY, Wan LL, Yang QJ, Han YL, Li D, Lu J, Guo C: Nilotinib reverses ABCB1/P-glycoprotein-mediated multidrug resistance but increases cardiotoxicity of doxorubicin in a MDR xenograft model. Toxicol Lett 2016;259:124-132.

9 Kee HJ, Park S, Kang W, Lim KS, Kim JH, Ahn Y, Jeong MH: Piceatannol attenuates cardiac hypertrophy in an animal model through regulation of the expression and binding of the transcription factor GATA binding factor 6 FEBS Lett 2014;588:1529-1536.

10 Obitsu S, Sakata K, Teshima R, Kondo K: Eleostearic acid induces RIP1-mediated atypical apoptosis in a kinase-independent manner via ERK phosphorylation, ROS generation and mitochondrial dysfunction. Cell Death Dis 2013;4:e674.

-11 Horikawa I, Fujita K, Jenkins LM, Hiyoshi Y, Mondal AM, Vojtesek B, Lane DP, Appella E, Harris CC: Autophagic degradation of the inhibitory p53 isoform $\Delta 133 \mathrm{p} 53 \alpha$ as a regulatory mechanism for p53mediated senescence. Nat Commun 2014;5:4706.

-12 Green DR, Llambi F: Cell Death Signaling. Cold Spring Harb Perspect Biol DOI: 10 1101/cshperspect. a006080.

13 Xia P, Liu Y, Cheng Z: Signaling Pathways in Cardiac Myocyte Apoptosis. Biomed Res Int 2016;2016:9583268.

14 Vakifahmetoglu-Norberg H, Ouchida AT, Norberg E: The role of mitochondria in metabolism and cell death. Biochem Biophys Res Commun 2017;482:426-431.

-15 Goldenthal MJ: Mitochondrial involvement in myocyte death and heart failure. Heart Fail Rev 2016;21:137155. 


\section{Cellular Physiology Cell Physiol Biochem 2017;42:2182-2193 \begin{tabular}{ll|l} 
DOI: 10.1159/000479993 & and Biochemistry Published online: August 15, 2017 & $\begin{array}{l}\text { O 2017 The Author(s). Published by S. Karger AG, Basel } \\
\text { www.karger.com/cpb }\end{array}$
\end{tabular}}

Yang et al.: Nilotinib Induced Caspase-Independent Signaling in H9C2

16 Song YH, Cai H, Zhao ZM, Chang WJ, Gu N, Cao SP, Wu ML: Icariin attenuated oxidative stress inducedcardiac apoptosis by mitochondria protection and ERK activation. Biomed Pharmacother 2016;83:10891094.

17 Guo R, Lin J, Xu W, Shen N, Mo L, Zhang C, Feng J: Hydrogen sulfide attenuates doxorubicin-induced cardiotoxicity by inhibition of the p38 MAPK pathway in H9c2 cells. Int J Mol Med 2013;31:644-650.

18 Sevrioukova IF: Apoptosis-inducing factor: structure, function, and redox regulation. Antioxid Redox Signal 2011;14:2545-2579.

19 Norberg E, Orrenius S, Zhivotovsky B: Mitochondrial regulation of cell death: processing of apoptosisinducing factor (AIF). Biochem Biophys Res Commun 2010;396:95-100.

-20 Ranjan A, Iwakuma T: Non-Canonical Cell Death Induced by p53 Int J Mol Sci DOI:103390/ ijms17122068.

21 Cregan SP, Dawson VL, Slack RS: Role of AIF in caspase-dependent and caspase-independent cell death. Oncogene 2004; 23:2785-2796.

22 Leist M, Jäättelä M: Triggering of apoptosis by cathepsins. Cell Death Differ 2001;8:324-326.

23 de Castro MA, Bunt G, Wouters FS: Cathepsin B launches an apoptotic exit effort upon cell death-associated disruption of lysosomes. Cell Death Discov 2016;2:16012.

24 Lin L, Baehrecke EH: Autophagy, cell death, and cancer. Mol Cell Oncol 2015;2:e985913.

25 Song S, Tan J, Miao Y, Li M, Zhang Q: Crosstalk of autophagy and apoptosis: Involvement of the dual role of autophagy under ER stress. J Cell Physiol DOI: 101002/jcp.25785.

-26 Dong Z, Liang S, Hu J, Jin W, Zhan Q Zhao K: Autophagy as a target for hematological malignancy therapy. Blood Rev 2016;30:369-380.

-27 Gottlieb RA, Mentzer RM: Autophagy during cardiac stress: joys and frustrations of autophagy. Annu Rev Physiol 2010;72:45-59.

28 Kobayashi S, Xu X, Chen K, Liang Q: Suppression of autophagy is protective in high glucose-induced cardiomyocyte injury. Autophagy 2012;8:577-592.

29 Dutta D, Xu J, Kim JS, Dunn WA, Jr., Leeuwenburgh C: Upregulated autophagy protects cardiomyocytes from oxidative stress-induced toxicity. Autophagy 2013;9:328-344.

-30 Wolf A, Couttet P, Dong M, Grenet O, Heron M, Junker U, Ledieu D, Mahl A, Marrer E, Persohn E, Pognan F, Zhou W, Tsao J, Roman D: Preclinical evaluation of potential nilotinib cardiotoxicity. Leuk Res 2011;35:631637.

-31 Moreira AC, Branco AF, Sampaio SF, Cunha-Oliveira T, Martins TR, Holy J, Oliveira PJ, Sardão VA: Mitochondrial apoptosis-inducing factor is involved in doxorubicin-induced toxicity on H9c2 cardiomyoblasts. Biochim Biophys Acta 2014;1842:2468-2478.

-32 Hsu SF1, Hsu CC, Cheng BC, Lin CH: Cathepsin B is involved in the heat shock induced cardiomyocytes apoptosis as well as the anti-apoptosis effect of HSP-70 Apoptosis 2014;19:1571-1580.

-33 Bhoopathi P, Chetty C, Gujrati M, Dinh DH, Rao JS, Lakka S: Cathepsin B facilitates autophagy-mediated apoptosis in SPARC overexpressed primitive neuroectodermal tumor cells. Cell Death Differ 2010;17:15291539.

34 Wang Y, Liu J, Tao Z, Wu P, Cheng W, Du Y, Zhou N, Ge Y, Yang Z: Exogenous HGF Prevents Cardiomyocytes from Apoptosis after Hypoxia via Up-Regulating Cell Autophagy. Cell Physiol Biochem 2016;38:24012413.

35 Zhou XY, Luo Y, Zhu YM, Liu ZH, Kent TA, Rong JG, Li W, Qiao SG, Li M, Ni Y, Ishidoh K, Zhang HL: Inhibition of autophagy blocks cathepsins-tBid-mitochondrial apoptotic signaling pathway via stabilization of lysosomal membrane in ischemic astrocytes. Cell Death Dis 2017;8:e2618. 